\title{
Bovinos Mestiços Alimentados com Diferentes Proporções de Volumoso:Concentrado. 1. Digestibilidade Aparente dos Nutrientes, Ganho de Peso e Conversão Alimentar ${ }^{1}$
}

\author{
Flávio Dutra de Resende ${ }^{2}$, Augusto César de Queiroz ${ }^{3}$, José Victor de Oliveira², \\ José Carlos Pereira ${ }^{3}$, Antonio Bento Mâncio ${ }^{3}$
}

\begin{abstract}
RESUMO - Os objetivos deste trabalho foram avaliar os efeitos da relação volumoso: concentrado sobre ganho de peso e conversão alimentar e determinar a digestibilidade aparente dos nutrientes de 25 e 20 novilhos mestiços, com $310 \mathrm{~kg}$ PV médio inicial, respectivamente. Cinco dietas foram fornecidas à vontade, à base de feno de capim-tanzânia e concentrado, em diferentes proporções de volumoso:concentrado (85:15; 70:30; 55:45; 40:60; e 25:75). A análise de regressão dos coeficientes de digestibilidade aparente da matéria seca, matéria orgânica, fibra em detergente neutro, proteína bruta e energia bruta, em função do nível de concentrado na dieta, apresentou efeito quadrático e os coeficientes máximos estimados foram de 67, 70, 51, 42, 74 e 69\%, para os níveis de 54, 58, 28, 12, 34 e 77,0\% de concentrado na dieta, respectivamente. O ganho médio diário de PV (GMD) elevou-se linearmente com o aumento do nível de concentrado na dieta. Observaram-se incrementos de $0,00895 \mathrm{~kg} /$ dia no GMD para cada aumento percentual do nível de concentrado na dieta, entre 15 e 75\%. Nesta mesma faixa, a conversão alimentar da MS, PB e EB melhorou 0,08; 0,011468; e 0,50646, respectivamente, para cada aumento percentual do nível de concentrado na dieta. A máxima digestão dos nutrientes para a máxima eficiência de utilização ocorreu com níveis de ingestão de FDN variando de 1,25 a 1,02\% PV para novilhos mestiços.
\end{abstract}

Palavras-chave: bovino de corte, ganho de peso, nível de concentrado, nutriente

\section{Feedlot Confined Crossbreed Steers Fed with Different Forage to Concentrate Ratios. 1. Apparent Nutrient Digestibilities, Weight Gain and Feed:Gain Ratio}

\begin{abstract}
The objective of this work was to evaluate the effects of the forage to concentrate ratio on the weight gain and feed: gain, and to determine the apparent digestibility of the nutrients of 25 and 20 crossbred steers averaging $310 \mathrm{~kg}$ LW, respectively. Five full fed diets based on tanzaniagrass hay and concentrate, with different forage to concentrate ratio $(85: 15,70: 30,55: 45 ; 40: 60 ;$ and $25: 75)$ were used. The regression analysis of the coefficienties of apparent digestible dry matter, digestible organic matter, neutral detergent fiber, digestible protein and gross energy in function of the concentrate level in the diets presented quadratic effect and the maximum estimated coefficienties were 67.0, 70.0, 51.0 42.074 .0 and $69.0 \%$, for the levels of 54, 58, 28, 12, 34 e $77 \%$ of concentrate in the diets, respectively. The average daily gain (ADG) linearly increased with the increase of the concentrate level in the diets. There was an increase of $0.00895 \mathrm{~kg} / \mathrm{day}$ in the ADG for each percent increase on the concentrate level in the diets, from 15.0 to $75.0 \%$. In the same range, the feed: gain of DM, CP, and GE increased 0.08; 0.011468; and 0,50646, respectively, for each percent increase on the concentrate level in the diets. The maximum nutrient digestion for the maximum efficiency of nutrient utilization occurred with the intake of neutral detergent fiber ranged from 1.25 to $1.02 \% \mathrm{LW}$ for crossbred steers
\end{abstract}

Key Words: average daily gain, beef cattle, concentrate level, digestibility, nutrient

\section{Introdução}

Estudos de como a relação volumoso:concentrado influi na cinética da digestão, bem como na utilização dos alimentos, são fundamentais para formular e manipular as dietas utilizadas, visando a máxima eficiência da mesma.

Segundo VAN SOEST (1994), a digestão pode ser definida como um processo de conversão de macromoléculas da dieta em compostos mais simples, que podem ser absorvidos a partir do trato gastrintestinal. Para esse autor, medidas de digestibilidade dos nutrientes servem para qualificar os alimentos quanto ao seu valor nutritivo. Estudos de digestão dos nutrientes são importantes para quantificar a absorção destes nos diferentes compartimentos do trato gastrintestinal, proporcionando condições mais adequadas de avaliação de dietas, bem como maior eficiência de uso da dieta pelo animal. Segundo COELHO DA SILVA e LEÃO (1979), a

\footnotetext{
1 Parte da tese de doutorado apresentada pelo primeiro autor, junto ao Depto. de Zootecnia da Universidade Federal de Viçosa.

2 Pesquisador Científico do Instituto de Zootecnia, Cx. Postal 35, 14770-000 - Colina - SP. E.mail: eezcol@colina.com.br

3 Professor do Departamento de Zootecnia da UFV, Viçosa - MG. E.mail: acqueiroz@mail.ufv.br
} 
digestibilidade dos nutrientes depende das características do alimento, e não do animal. Segundo McDONALD et al. (1993), existem vários fatores que influenciam a digestibilidade: composição dos alimentos e da ração; preparo dos alimentos; fatores dependentes dos animais e do nível nutricional, entre outros. A estimativa da quantidade de matéria seca digerida nos diversos compartimentos do trato gastrintestinal é de grande importância, uma vez que, de posse desses valores, pode-se conhecer o local de absorção dos demais nutrientes.

Vários estudos têm sido conduzidos para discutir os efeitos na digestibilidade total e parcial dos nutrientes influenciados por diferentes níveis de concentrado na dieta. DUCKWORTH (1946), estudando a influência da fibra bruta sobre a digestibilidade de forragens, verificou que o conteúdo de fibra na dieta foi o maior depressor da digestibilidade. A cada aumento de $1 \%$ no conteúdo de fibra, ocorreu queda de 0,9 e $0,5 \%$, na digestibilidade, para taurinos e zebuínos, respectivamente.

CHURCH (1988) relata que há decréscimo de $4 \%$ na digestibilidade para cada aumento de $1 \%$ no nível de ingestão acima da mantença. Dependendo das condições, a digestibilidade dos ingredientes das rações pode ser aditiva ou substitutiva, podendo ocorrer efeitos associativos negativos. Joanning et al., citados por CHURCH (1988), não notaram efeito associativo negativo na digestibilidade de rações com mistura de silagem de milho e grão, quando fornecidas em níveis menores que duas vezes a mantença; porém observaram decréscimo de $11 \%$ na digestibilidade da matéria seca (MS), quando esta ração foi fornecida de 2,4 a 3,1 vezes a mantença. Em dietas compostas por grãos em alta proporção, 50\% do amido e $30 \%$ da fibra podem ser digeridos pósrúmen. Mudanças no local de digestão são, em grande parte, ditadas por aumento na taxa de passagem e possível decréscimo taxa de digestão ruminal (CHURCH, 1988).

MILLER e MUNTIFERING (1985), avaliando o efeito dos níveis de concentrado de 0, 20, 40,60 e 80\% sobre as características cinéticas que influenciam a digestão da fibra do volumoso in vivo, verificaram que o potencial de degradação da fibra in situ foi o determinante primário da depressão de sua digestibilidade, quando o nível de concentrado na dieta aumentou. Observaram, ainda, que o potencial da digestão da fibra do volumoso foi mais baixo para o nível de $80 \%$ de grãos na dieta, comparado aos outros tratamentos, indicando que a fermentação de grãos não prejudica, significativamente, o potencial de digestão da fibra até $60 \%$ de inclusão desses na dieta. Para esses autores, a análise da cinética da digestão pode ser útil para explicar a depressão na digestibilidade, mostrando que esses fatores, no processo de digestão, são influenciados mais adversamente quando se utilizam grãos nas rações. Além do efeito do $\mathrm{pH}$, a competição entre a digestão e a passagem para uma fibra potencialmente digestível pode variar a digestibilidade da fibra, quando concentrados são utilizados nas rações.

Em face do exposto, o objetivo deste trabalho foi estudar os efeitos de diferentes proporções volumoso:concentrado, utilizando-se cinco níveis de fibra em detergente neutro, sobre o ganho de peso e a conversão alimentar e digestibilidade aparente dos nutrientes, de bovinos mestiços alimentados em regime de confinamento.

\section{Material e Métodos}

O presente estudo foi realizado na Estação Experimental de Zootecnia de Colina, em Colina - SP, unidade pertencente ao Instituto de Zootecnia do Estado de São Paulo em duas etapas. Na primeira, para medir o desempenho, foram utilizados 25 bovinos mestiços machos (5/8 europeu/zebu), castrados aos sete meses, com peso vivo médio inicial de $310 \mathrm{~kg}$ e, aproximadamente, 24 meses de idade, mantidos confinados em baias individuais com piso cimentado, medindo $4 \times 6$, sendo $2 / 3$ da área cobertos e o restante, $1 / 3$, descobertos (solário). As baias eram providas de comedouro e bebedouro individuais. Na segunda etapa, para determinar a digestibilidade aparente dos nutrientes, foram utilizados 20 animais semelhantes aos da primeira etapa, mantidos em regime de confinamento (fase de adaptação) e, em seguida, foram colocados em gaiolas de metabolismo confeccionadas em madeira, dotadas de comedouro e bebedouro individuais, e reguláveis em função do tamanho do animal. Foi utilizada as mesmas rações contidas em RESENDE et al. (2001). Nas duas etapas experimentais, os animais passaram por um período de adaptação às instalações e dietas experimentais de 28 dias, dando início à fase experimental de 84 dias (primeira etapa) e sete dias (segunda etapa). Finalizada a adaptação dos animais, estes foram pesados no início e final das fases experimentais, após jejum prévio de 16 horas. No período experimental, mediu-se a ração fornecida e as sobras deixadas pelos animais diariamente.

$\mathrm{Na}$ etapa um, as rações foram fornecidas à 
Rev. bras. zootec.

vontade, individualmente, conforme o tratamento, duas vezes/dia, às 8 e $16 \mathrm{~h}$, controlando-se a ingestão durante todo o período de coleta, procurando-se manter as sobras entre 5 e $10 \%$ do total fornecido. Diariamente, pela manhã, antecedendo ao fornecimento das rações, retiraram-se as sobras, pesando-as e anotando-se os dados em planilhas apropriadas de controle diário de fornecido e sobras. Na etapa dois, para medir a ingestão voluntária dos animais, adotaram-se os mesmos critérios da etapa um, do dia $21^{\circ}$ ao 28 음 da fase de adaptação. A partir daí, na fase de coleta, forneceu-se $90 \%$ da ingestão obtida do $21^{\circ}$ ao $28^{\circ}$ dia, duas vezes/dia, medindo-se eventuais sobras que pudessem ocorrer. Nas duas etapas, as sobras foram então amostradas na proporção de $5 \%$ do total das mesmas, acondicionadas em sacolas plásticas devidamente etiquetadas e armazenadas em freezer. Semanalmente, amostraram-se o feno fornecido e os diferentes concentrados utilizados, acondicionando-se as amostras em sacolas plásticas e guardadas em freezer. Ao final do período experimental, as amostras de feno e concentrados fornecidos, bem como as de sobras de cada animal, foram retiradas do freezer, descongeladas em temperatura ambiente e homogeneizadas manualmente. Para cada animal fez-se uma amostra composta das sobras do período, secando-a em estufa de ventilação forçada a $60 \pm 5^{\circ} \mathrm{C}$, por 48 horas, para determinação da amostra pré-seca (ASA). Da mesma forma, procedeu-se com o feno e concentrados fornecidos. Posteriormente, as mesmas foram moídas em moinho tipo "Willey", com peneira de 30 "mesh", acondiocionadas em vidro para posteriores análises químicobromatológicas. Na segunda etapa, as fezes foram coletadas 48 horas após o engaiolamento dos animais, em caixotes de madeira, revestidos internamente com borracha adaptados na parte posterior das gaiolas. As fezes produzidas/animal/dia (gaiola de metabolismo) foram também pesadas e amostradas diariamente. Somente $5 \%$ do total das sobras e das fezes produzidas/animal/dia foram acondicionadas em sacolas plásticas e congeladas a $-18^{\circ} \mathrm{C}$, sendo posteriormente composta numa amostra/animal e processada da mesma forma que as sobras e alimento fornecido.

As análises químicas foram realizadas no Laboratório de Nutrição Animal do Departamento de Zootecnia da Universidade Federal de Viçosa e na Estação Experimental de Zootecnia de Colina. Realizaram-se as determinações de matéria seca, fibra em detergente neutro, fibra em detergente ácido, proteína bruta, energia bruta e matéria orgânica, conforme metodologias descritas por SILVA (1990).

Usou-se o delineamento experimental inteiramente casualizado com cinco tratamentos e cinco repetições (primeira etapa) e cinco tratamentos e quatro repetições (segunda etapa). As análises estatísticas das variáveis estudadas foram interpretadas por análise de variância e de regressão, utilizando-se o programa (SAS, 1992) Statistical Analysis Systems e os coeficientes de regressão foram comparados pelo teste $\mathrm{t}$, a $5 \%$ de probabilidade.

\section{Resultados e Discussão}

As equações de regressão ajustadas e os coeficientes de digestibilidade aparente da matéria seca (CDAMS), matéria orgânica (CDAMO), fibra em detergente neutro (CDAFDN), fibra em detergente ácido (CDAFDA), proteína bruta (CDAPB) e energia bruta (CDAEB) das diferentes dietas experimentais, em função dos níveis de concentrado utilizados na dieta, são mostrados na Tabela 1 .

As análises de regressão dos CDAMS, CDAMO, CDAFDN, CDAFDA, CDAPB e CDAEB, em função do teor de concentrado da dieta, revelaram efeito quadrático $(\mathrm{P}<0,01)$ do teor de concentrado sobre os coeficientes de digestibilidade das variáveis estudadas. Derivando as equações de regressão obtidas, obteve-se o ponto máximo para os coeficientes de digestibilidade aparente da MS, MO, FDN, FDA, PB e EB de 67, 70, 51, 42, 74 e 69\%, respectivamente, correspondente à utilização de 54, 58, 28, 12, $34 \mathrm{e}$ $77 \%$ de concentrado na dieta, respectivamente. A variação da estimativa dos coeficientes de digestibilidade aparente da MS, FDN e FDA para dos diferentes níveis de concentrados é mostrada na Figura 1.

Os valores obtidos para CDAMS e CDAMO concordam com os resultados obtidos por VALADARES FILHO el al. (1987) e OLIVEIRA et al. (1994). Segundo VALADARES FILHO et al. (1987), carboidratos não-estruturais possuem coeficiente de digestibilidade aparente total acima de $90 \%$ e carboidratos estruturais próximos de $50 \%$, o que reflete na maior digestão da MS nas rações com menores teores de carboidratos estruturais de maior teor de concentrado.

Conclusão semelhante obteve RODE et al. (1985), que utilizaram diferentes níveis de concentrado na dieta, observando que os CDAMS e CDAMO elevaram-se, à medida que aumentou o concentrado na dieta, provavelmente em virtude da redução de 
Tabela 1 - Médias e equações de regressão ajustadas (ER) para coeficiente de digestibilidade aparente da matéria seca (CDAMS), matéria orgânica (CDAMO), fibra em detergente neutro (CDAFDN), fibra em detergente ácido (CDAFDA), proteína bruta (CDAPB) e energia bruta (CDAEB), em função dos níveis de concentrado na dieta (c)

Table 1 - Means and fitted regression equations (RE) for the coefficienties of apparent digestible dry matter (CADDM), digestible organic matter (CADOM), neutral detergent fiber (CADNDF) and acid detergent fiber (CADADF), digestible protein (CDCP) and gross energy (CAGE), in function of the concentrate levels in the diet

\begin{tabular}{lccccccc}
\hline Item & \multicolumn{9}{c}{$\begin{array}{c}\text { Nível de concentrado na dieta } \\
\text { Concentrate level in the diet }\end{array}$} \\
\cline { 2 - 7 } & 15 & 30 & 45 & 60 & 75 & EV \\
$(R E)$
\end{tabular}

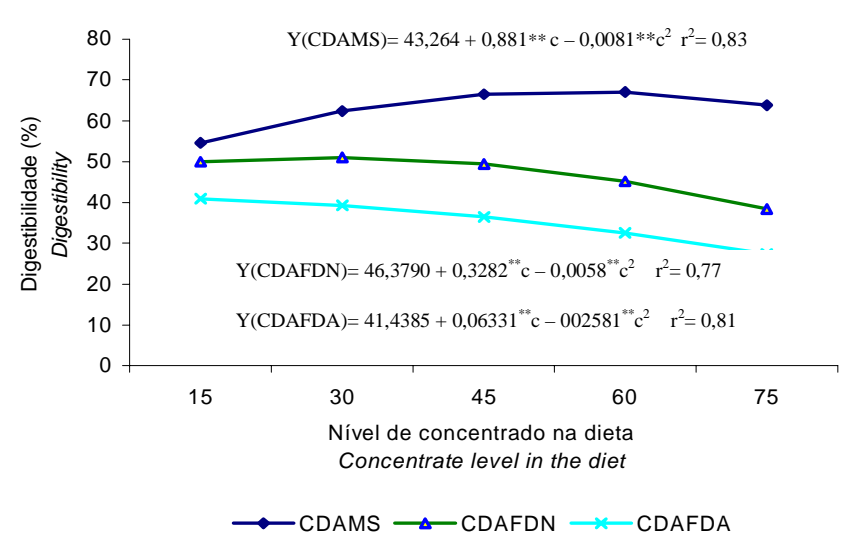

Figura 1 - Estimativa dos coeficientes de digestibilidade aparente da matéria seca (CDAMS), fibra em detergente neutro (CDAFDN) e fibra em detergente ácido (CDAFDA), em função dos níveis de concentrados na dieta.

Figure 1 - Estimate of coefficients of apparent digestibility of dry matter (CADDM), neutral detergent fiber (CADNDF) and acid detergent fiber (CADADF) in function of the concentrate levels in the diet. carboidratos estruturais e do aumento no teor de carboidratos não-estruturais na dieta.

CARVALHO et al. (1997) verificaram que houve efeito quadrático para as digestibilidades aparentes da MS, MO e dos carboidratos totais (CHT), estimando as digestibilidades máximas desses nutrientes aos níveis de 42, 40 e $43 \%$ de concentrado nas rações totais, respectivamente. RODRIGUES et al. (1996) também verificaram maior digestibilidade da MS, com o aumento do nível de concentrado na dieta. DUTRA et al. (1997) encontraram para CDAMS e CDAMO maiores valores para a ração com baixa fibra em relação à de alta fibra $(52,2$ vs $38,7 \%$ e 54,7 vs $42,0 \%$, respectivamente), o que foi atribuído à maior presença de carboidratos totais digestíveis em relação aos carboidratos estruturais contidos na ração de baixa fibra. Comparando níveis de 20 e $60 \%$ de concentrado na dieta, BERCHIELLI (1994) obteve maiores valores CDAMS, 50,5 e 61,3\% e CDAMO de 52,0 e $62,8 \%$, respectivamente. 
ARAÚJO et al. (1998), avaliando dietas com níveis de concentrado variando de 10 a 90\%, também verificaram influência destes na digestibilidade aparente da MS, MO, PB, FDN e FDA. Esses autores verificaram que o CDAMS e o CDAMO aumentou linearmente, à medida que os níveis de concentrado na dieta variaram de 10 para $90 \%$, tendo os valores médios obtidos variando de 53,5 a 73,0 e 55,0 a 74,1, respectivamente. BÜRGER et al. (2000) também verificaram efeito linear crescente nos CDAMS e CDAMO ao variar os níveis de concentrado na dieta de 30 a $90 \%$. Outros autores verificaram efeito linear positivo do CDAMS e CDAMO com o aumento dos níveis de concentrado na dieta (ATWELL et al., 1991 e HATENDI et al., 1996).

Os aumentos verificados no coeficiente de digestibilidade aparente da EB, com a elevação do teor concentrado na dieta, no presente estudo, estão de acordo com os resultados obtidos por LIMA (1986), que observou incrementos no coeficiente de digestibilidade da EB, ao aumentar o nível de concentrado na dieta. Também, GOMES et al. (1994), LORENZONI et al. (1986), RESENDE (1984), MORAN (1985) e RODRIGUES et al. (1996) reportaram aumentos similares na digestibilidade da EB, ao utilizarem rações mais concentradas.

A correlação entre a ingestão voluntária de MS e a digestibilidade da dieta feita por CONRAD et al. (1964) mostrou que, à medida que a digestibilidade se elevavou de 52 até $67 \%$, houve aumento na ingestão. $\mathrm{O}$ aumento da digestibilidade acima desse limite resultou em decréscimo da ingestão, e, a partir daí, a ingestão de energia digestível manteve-se constante, passando a ingestão a ser regulada por mecanismos quimiotásticos. Dessa forma, CONRAD et al. (1964), concluíram que a ingestão e a digestibilidade podem estar positiva ou negativamente correlacionadas entre si, dependendo da qualidade da dieta. A correlação é positiva, quando se utilizam dietas de baixa qualidade, pois o volume ocupado pela fração de baixa digestibilidade reduz a ingestão. Por outro lado, a ingestão e a digestibilidade são negativamente correlacionadas, quando se trata de dietas de alta qualidade, em que a fração fibrosa é pequena e, provavelmente, não afeta a ingestão e esta será controlada pelo requerimento energético do animal. Neste estudo, os CDAMS e CDAMO máximos foram obtidos, ao se utilizar 54 e $58 \%$ de concentrado na dieta, respectivamente, níveis estes próximos aos níveis de concentrado na dieta que otimizou a ingestão de energia digestível, em $\mathrm{kcal} / \mathrm{kg}^{0,75}$ com $57 \%$ de concentrado e Mcal/dia com $62 \%$ de concentrado (RESENDE et. al, 2001). Com base nestes níveis de concentrado obtidos, 54 e $58 \%$ de concentrado na dieta, e utilizando-se a equação de regressão obtida por RESENDE et al. (2001) para ingestão de FDN (Y $\left.=2,0178-0,01605 c, \mathrm{r}^{2}=0,92\right)$, em que $\mathrm{Y}$ é ingestão estimada de FDN, em \%PV, e $c$, níveis de concentrado na dieta, verificou-se que a ingestão de FDN, em $\% \mathrm{PV}$, que maximizou os CDAMS e CDAMO, foi de 1,15 e $1,09 \%$, respectivamente, resultados estes que se encontram na faixa de ingestão ótima de FDN para bovinos leiteiros, recomendada pelo NRC (1989), de $1,2 \pm 0,1 \% \mathrm{PV}$.

Os CDAMS e CDAMO máximos obtidos foram 67 e $70 \%$, respectivamente, resultados estes próximos ao obtido por CONRAD (1964), de 67\%. Porém, MONTGOMERY e BAUMGARDT (1965) observaram que a máxima ingestão de energia foi obtida com CDAMS de $56 \%$, o que denota, provavelmente, não haver um ponto fixo de digestibilidade que regula o limite de transição entre o controle físico e fisiológico da ingestão voluntária. Além das diferenças entre as dietas, o ponto de transição dependerá da demanda energética do animal.

No caso da FDN e da FDA, observou-se, após derivações das regressão obtidas, que a digestibilidade máxima destes ocorreu, quando se usou 28 e $12 \%$ de concentrado na dieta, respectivamente, e, a partir daí, os aumentos no teor de concentrado na dieta resultaram em decréscimo na sua digestibilidade, o que era de se esperar, uma vez que, com a elevação do concentrado na dieta, há efeito depressor deste sobre a digestibilidade da fibra (Figura 1). ARAÚJO et al. (1998) também verificaram decréscimo linear na digestibilidade da FDN, com o aumento dos níveis de concentrado na dieta de 10 a $90 \%$, tendo os valores médios da digestibilidade da FDN variado de 50,8 a $29,4 \%$, respectivamente. Já BÜRGER et al. (2000) não verificaram influência dos níveis de concentrado na dieta sobre digestibilidade da FDN, tendo obtido o valor médio de 49,3\%; fato confirmado por DUTRA et al. (1997) e BERCHIELI (1994), que também não observaram efeitos dos níveis de concentrado sobre a digestibilidade da FDN, encontrando valores médios de 21,7 e $44,1 \%$, respectivamente. RODRIGUES et al. (1996) verificaram maior digestibilidade da FDN para a dieta que continha $12,5 \%$ de concentrado, não havendo diferenças entre as dietas com 25,0, 37,5 e $50,0 \%$ de concentrado.

Para a FDA, ARAÚJO et al. (1998) observaram efeito quadrático dos níveis de concentrado sobre a 
digestibilidade da FDA, sendo o valor máximo obtido quando se utilizou $29 \%$ de concentrado na dieta, porém RODRIGUES et al. (1996) não observaram diferenças na digestibilidade da FDA, sendo o valor médio obtido de $49,5 \%$.

Verificou-se efeito quadrático dos níveis de concentrado sobre os coeficientes de digestibilidade aparente da proteína bruta (CDAPB) (Tabela 1). A maior digestibilidade aparente da $\mathrm{PB}$, à medida que se elevou o teor de concentrado na dieta, foi, provavelmente, reflexo da maior ingestão de proteína, que reduz a importância relativa das perdas endógenas, elevando os valores de digestibilidade aparente. RODRIGUES et al. (1996) não encontraram diferenças no CDAPB, ao utilizaram variados os níveis de concentrado na dieta de 12,5 a 50,0\%, encontrando valor médio de $66 \%$, o mesmo sendo observado por ANDRADE (1992) e OLIVEIRA et al. (1994), que também não encontraram diferenças no CDAPB, em resposta à elevação dos teores de concentrado nas dietas utilizadas. Já LIMA (1986) encontrou maiores CDAPB para a ração com maior proporção de concentrado. CARVALHO et al. (1997) verificaram que os níveis de concentrado na dieta não afetaram a digestibilidade da $\mathrm{PB}$, encontrando valores médios de $63,5 \%$.

O ganho médio diário de peso vivo (GMDPV) e a conversão alimentar da matéria seca (CAMS), proteína bruta (CAPB) e energia bruta (CAEB) dos animais nos diversos tratamentos estão mostrados no Tabela 2.

Verificou-se efeito linear positivo $(\mathrm{P}<0,05)$ do nível de concentrado na dieta sobre o ganho médio diário de peso vivo ( $\mathrm{kg} / \mathrm{dia}$ ) e negativo sobre a melhoria da conversão alimentar da matéria seca (kg de MS ingerida/kg de ganho de peso vivo), da proteína bruta (kg de PB ingerida/ kg de ganho de peso vivo) e da energia bruta (Mcal de EB ingerida/ kg de ganho de peso vivo).

Segundo ROHR e DAENICKE (1984), o GMDPV é uma medida indispensável para se estimar o desenvolvimento do animal nos processos alimentares e sistemas de produção. Diversos trabalhos têm demonstrado melhorias no desempenho animal com a inclusão de concentrado na dieta. Segundo PRESTON e WILLIS (1974) e Levy et al. (1975) e Bartle et al. (1994), citados por FEIJÓ et al. (1996), encontramse, na literatura, resultados mostrando que o ganho de peso foi maior em animais alimentados com dietas ricas em concentrado. Segundo BOND et al. (1972), a velocidade de crescimento também foi conseqüência da maior quantidade de energia ingerida pelos animais que se alimentaram de dietas constituídas basicamente de concentrado, quando comparadas àquelas fornecidas a animais que ingeriram dietas mistas (feno + concentrado) ou dietas com apenas feno.

WOODY et al. (1983), trabalhando com novilhos alimentados com uma dieta à base de silagem de milho contendo $50 \%$ de grãos, verificaram que os mesmos ganharam mais ( $1,02 \mathrm{vs} 0,83 \mathrm{~kg} / \mathrm{dia})$ e foram mais eficientes ( $8,33 \mathrm{vs} 9,74 \mathrm{~kg} \mathrm{MS} / \mathrm{kg}$ ganho) que os alimentados com silagem contendo $29 \%$ de grãos. Quando o total de grãos na dieta aumentou de 55 para $96 \%$, os animais tiveram altas taxas de ganho diário $(1,22 \mathrm{vs} 1,06 \mathrm{~kg})$ e melhoraram a conversão alimentar (6,10 vs 8,50 kg MS/kg ganho). RIBEIRO (1997),

Tabela 2 - Médias e equações de regressão ajustadas (ER) para ganho médio diário (GMD), e conversão alimentar da matéria seca (CAMS), da proteína bruta (CAPB), e da energia bruta (CAEB), em função dos diferentes níveis de concentrados (c) na dieta.

Table 2 - Means and fitted regression equations (RE) for average weight gain (AWG), kg/day, and feed:gain ratio (FGDM), of crude protein $(F G C P)$, and of the gross energy (FGGE), in function of the different concentrate (c) levels in the diet

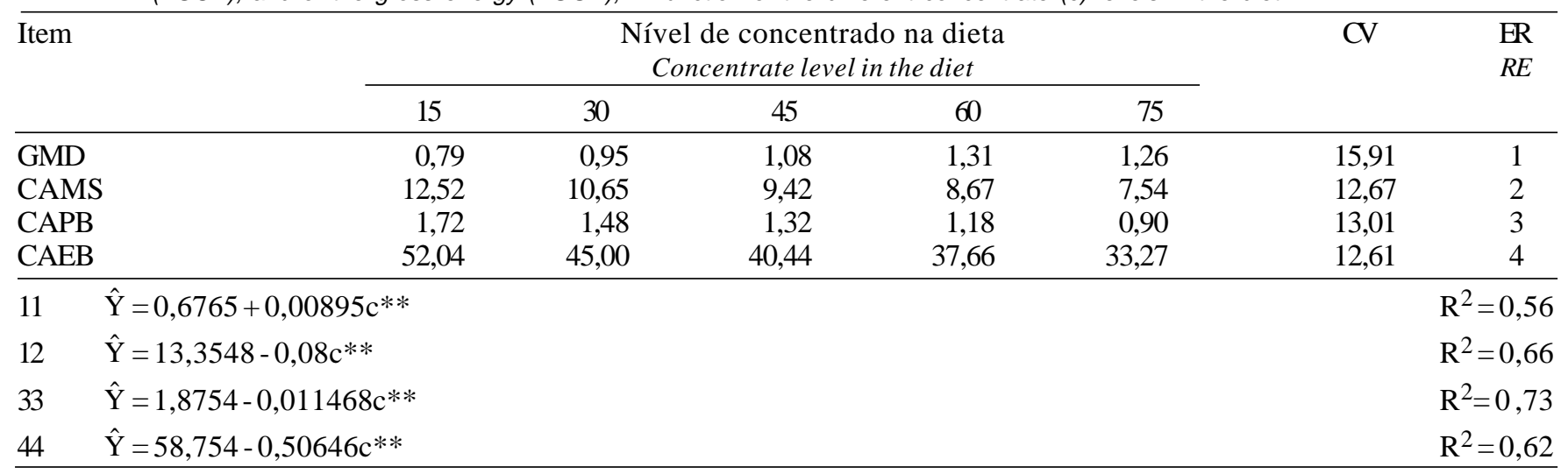


Rev. bras. zootec.

trabalhando com bezerros holandeses, alimentados com feno de coastcross picado mais concentrado em quatro proporções (45, 60, 75 e 90\%), verificou que o ganho médio diário de peso vivo aumentou linearmente com a elevação dos níveis de concentrado na dieta. Também HIRONAKA et al. (1994), trabalhando com novilhos Hereford, com peso inicial de $218 \mathrm{~kg}$, observaram acréscimo no ganho médio diário com o aumento do concentrado na dieta até um limite de $65 \%$. Acima deste nível, não houve incremento no ganho de peso. No presente experimento, animais alimentados com $75 \%$ de concentrado ganharam $37,3 \%$ mais peso $(\mathrm{kg} / \mathrm{dia})$ que os animais alimentados com $15 \%$ de concentrado (1,26 vs $0,79 \mathrm{~kg} / \mathrm{dia})$ e requereram $39,8 \%$ menos alimentos por unidade de ganho $(7,54$ vs 12,52$)$ nesta mesma faixa.

A análise de regressão dos dados entre os níveis de concentrado na dieta de 15 a $75 \%$ indicaram que o ganho médio diário (GMD) aumentou $0,00895 \mathrm{~kg}$ e a CAMS melhorou $0,08 \mathrm{~kg}$ MS/ $\mathrm{kg}$ ganho, para cada aumento percentual no nível de concentrado na dieta. $\mathrm{O}$ mesmo foi observado para CAPB e CAEB, que melhoraram 0,011468kg PB e 0,50646 Mcal EB ingerida/kg ganho, respectivamente. WOODY et al. (1983), trabalhando com níveis de 30 a $70 \%$ de concentrado na dieta, observaram valor de $0,009 \mathrm{~kg}$ de acréscimo no GMD para cada aumento percentual no nível de concentrado na dieta, resultado semelhante ao deste estudo. Observaram, também, melhora 0,058kg MS ingerida/kg ganho na CAMS.

Outros trabalhos também verificaram o impacto do aumento do nível de concentrado na dieta sobre o desempenho de animais confinados. Autores citados por WOODY et al. (1983), encontraram 0,006 e $-0,052$ (Peterson et al., 1973), 0,005 e -0,052 (Gill et al., 1976), 0,005 e -0,039 (Newland, 1976), 0,007 e -0,050 (Danner et al., 1980), 0,007 e-0,047 (Goodrich et al., 1974), de aumento no GMD e melhora na conversão alimentar, respectivamente, para cada aumento percentual do nível de concentrado na dieta. Entretanto, RIBEIRO (1997) não verificaram influência dos níveis de concentrado na dieta sobre a CAMS, embora tenha havido tendência em melhorar CAMS, à medida que onível dietético de concentrado aumentou.

Observa-se, na Figura 2, que a ingestão de MS, em kg/dia, permaneceu constante, de 10,09 kg/dia, quando a porcentagem de concentrado na dieta aumentou de 15 para $75 \%$ (RESENDE et. al, 2001). Nesse intervalo, o GMDPV aumentou linearmente, $0,00895 \mathrm{~kg} /$ dia para cada unidade percentual de acréscimo nos níveis de concentrado na dieta, o que

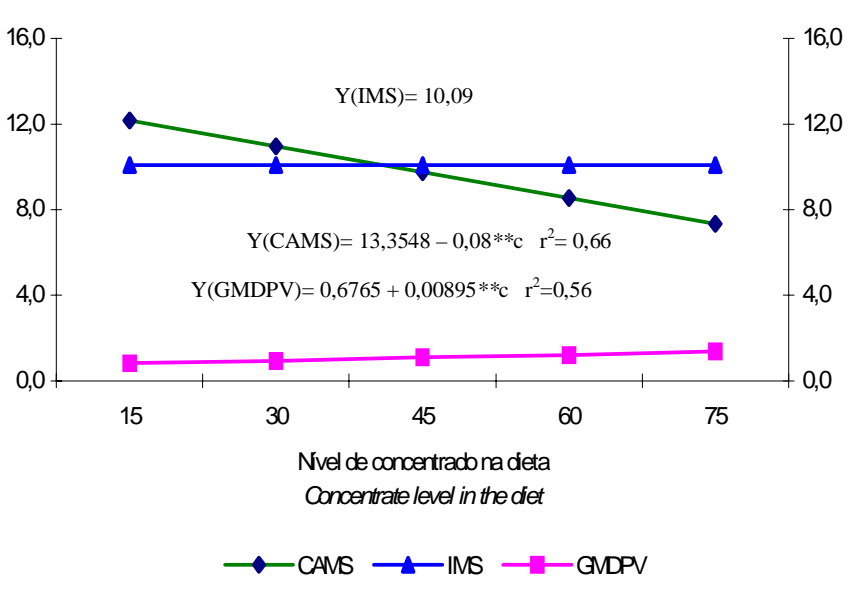

Figura 2 - Estimativa do ganho médio diário do peso vivo (GMDPV), em kg/dia, da conversão alimentar da matéria seca (CAMS), em $\mathrm{kg}$ MS ingerida/kg de ganho de peso vivo, e da ingestão de matéria seca (IMS), em função dos níveis de concentrado na dieta.

Figure 2 - Estimate of daily weight gain of live weight (DWGLV), $\mathrm{kg} /$ day, feed: gain ratio of dry matter (F:GDM), in $\mathrm{kg}$ ingested DM / $\mathrm{kg}$ of gain, of live weight and of dry matter intake (DMI) in function of concentrate levels in the diet.

demonstra a melhor eficiência de conversão em dietas de melhor qualidade. No presente estudo, requereu-se $-0,08 \mathrm{~kg}$ de MS ingerida para cada $\mathrm{kg}$ de ganho de peso, em função do aumento de uma unidade percentual de concentrado na dieta, entre 15 e $75 \%$.

Segundo o NRC (1984), a eficiência de utilização dos nutrientes da dieta para ganho de peso depende da concentração energética da ração, ou seja, da relação volumoso:concentrado, aumentando a eficiência, à medida que aumenta a proporção de concentrado na dieta. Dados do NRC (1984) mostram que rações com baixa concentração energética e elevada proporção de volumoso, contendo em média $16 \%$ de concentrado, são utilizadas com eficiência de $30 \%$ para ganho de peso, ao contrário de rações com elevada concentração energética, contendo em média $83 \%$ de concentrado, que são utilizadas com eficiência de $45,8 \%$ para o ganho de peso. Isso explica, em parte, a melhoria no desempenho dos animais, à medida que aumentou a quantidade de concentrado na dieta.

Embora tenha sido obtida relação linear entre o GMDPV e níveis de concentrado na dieta, observa-se, na Tabela 2, que houve tendência de 


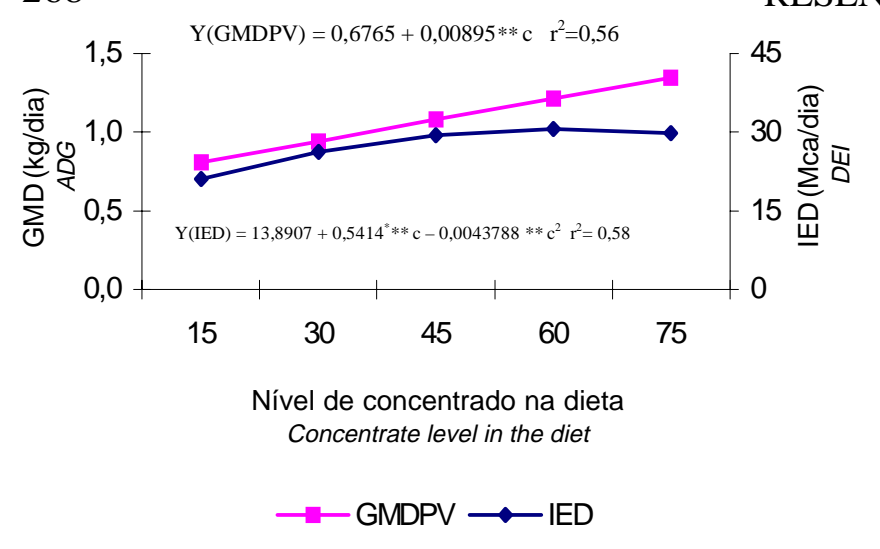

Figura 3 - Estimativa do ganho de peso (GMDV) e da ingestão de energia digestível (IED)(Mcal/dia) em função dos cinco níveis de concentrados na dieta.

Figure 3 - Estimate of weight gain (ADGLW) and digestible energy intake (DEI)(Mcal/day) in function of five concentrate levels in the diet.

redução no ganho, ao se utilizar $75 \%$ de concentrado, quando comparado com o nível de $60 \%$ de concentrado na dieta. Segundo Bernadon et al. e Vieira (1994), citados por FEIJÓ et al. (1996) e HIRONAKA et al. (1994), a resposta animal à adição de concentrado à dieta, é curvilínea e não-linear. Analisando a Figura 3, percebe-se que, embora o GMDPV seja linear, em função do aumento nos níveis de concentrado na dieta, a otimização da ingestão de energia digestível, expressa em Mcal/dia, foi obtida ao se utilizar $62 \%$ de concentrado na dieta (RESENDE et al., 2001), sugerindo que a melhor relação volumoso:concentrado utilizada no presente estudo foi de 40:60. Níveis de concentrado na dieta acima de $60 \%$ levaram à depressão na digestibilidade aparente da MS. Isto concorda com as observações de FOX e BLACK (1984), os quais relataram que, na utilização de altos níveis de concentrado na dieta, o GMDPV poderá ser influenciado em função da rápida queda na ingestão diária de $\mathrm{MS}$, o que ocorreu neste estudo, ao se utilizar 75\% de concentrado na dieta. Assim, segundo PRESTON e WILLIS (1974), o nível ótimo de concentrado na dieta é variável e tem como fatores determinantes o sexo, raça e idade do animal, qualidade do volumoso e concentrado, entre outros.

Como um dos principais objetivos na formulação de rações para ruminantes é fornecer-lhes a quantidade de nutrientes que resulte no melhor desempenho, pode-se concluir que a melhor relação volumoso:concentrado obtido no presente estudo, neste caso, foi 40:60. Neste ponto, maximizou-se o GMDPV com o máximo de volumoso na dieta. Uso de níveis de concentrado na dieta, acima de 60\%, não parece recomendável, devido ao aumento no custo de produção aliado ao maior gasto de concentrado na dieta e à incidência de transtornos metabólicos que podem ocorrer.

\section{Conclusões}

Houve efeito associativo positivo sobre os coeficientes de digestibilidade aparente da MS, MO, FDN, FDA, PB e EB até o limite de inclusão de 54, 58, 28, 12,37 e $77 \%$ de concentrado na dieta, respectivamente. A partir daí, verificou-se efeito depressor dos níveis de concentrado sobre a digestibilidade aparente destes nutrientes.

A maximização dos coeficientes de digestibilidade aparente da MS e MO foi obtida com um nível de ingestão de FDN de 1,15 e 1,09\% PV, respectivamente, estando estes, na mesma faixa recomendada para ingestão de FDN por bovinos leiteiros, de 1,2 $\pm 0,1 \% \mathrm{PV}$

$\mathrm{O}$ ganho médio diário de peso vivo (GMDPV) aumentou com a elevação dos níveis de concentrado na dieta, o mesmo sendo observado na melhoria da conversão alimentar da matéria seca, proteína bruta e energia bruta. A inclusão de elevada proporção de volumoso na dieta proporcionou boas taxas de GMDPV, embora, neste caso, a eficiência de utilização da MS, PB e EB tenha piorado.

\section{Referências Bibliográficas}

ANDRADE, A.T. Digestão total e parcial da matéria seca, matéria orgânica, energia bruta e proteína bruta em diferentes grupos genéticos de bovídeos. Viçosa, MG: UFV, 1992. 181p. Tese (Doutorado em Zootecnia) - Universidade Federal de Viçosa, 1992.

ARAÚJO, G.G.L., COELHO DA SILVA, J.F., VALADARES FILHO, S.C. et al. 1998. Consumo e digestibilidade total dos nutrientes de dietas contendo diferentes níveis de volumoso, em bezerros. Rev. Bras. Zootec., 27(2):345-354.

ATWELL, D.G., MERCHEN, N.R., JASTER, E.H. et al. 1991. Intake, digestibility and in situ kinectics of treated wheat straw and alfafa mixtures fed to holstein heifers. J. Dairy Sci., 74(10):3524-3534.

BERCHIELLI, T.T. Efeito da relação volumoso: concentrado sobre a partição da digestão, a síntese de proteína microbiana, produção de ácidos graxos voláteis e o desempenho de novilhos em confinamento. Belo Horizonte, MG: UFMG, 1994. 104p. Tese (Doutorado em Ciência Animal) Universidade Federal de Minas Gerais, 1994.

BOND, J., HOOVEN JR., N.W., WARICK, E. et al. 1972. Influence of breed and plane of nutrition on performance of dairy, dual-purpose and beef steers. II. From 180 days of age to slaughter. J. Anim. Sci., 34(6):1046-1053.

BÜRGER, P.J., PEREIRA, J.C., COELHO DA SILVA, J.F. et. al. 2000. Consumo e digestibilidade aparente total e parcial em bezerros holandeses alimentados com dietas contendo diferentes níveis de concentrado. Rev. bras. zootec., 29(1):206-214. 
Rev. bras. zootec.

CARVALHO, A.U., VALADARES FILHO, S.C., COELHO DA SILVA, J.F. et al. 1997. Níveis de concentrado em dietas de zebuínos. 1. Consumo e digestibilidade. Rev. bras. zootec., 26(5):986-995.

CHURCH, D.C. 1988. The ruminant animal digestive physiology and nutrition. xed. Englewood Cliffs: O \& Books Inc. 564p.

COELHO DA SILVA, J.F., LEÃO, M.I. 1979. Fundamentos da nutrição de ruminantes. 1.ed. Piracicaba: Livroceres. 380p.

CONRAD, H.R., PRATT, A.D., HIBBS, J.W. 1964. Regulation of feed intake in dairy cows. I - Change in importance of physical and physiological factors with increasing digestibility. J. Dairy Sci., 47(1):54-62.

DUCKWORTH, J. 1946. A statistical comparison on the influence of crude fibre on the digestibility of roughage by Bos indicus (Zebu) and Bos taurus cattle. Trop. Agric., 23(1):4-8.

DUTRA, A.R., QUEIROZ, A.C., PEREIRA, J.C. et al. 1997. Efeitos dos níveis de fibra e das fontes de proteínas sobre o consumo e digestão dos nutrientes em novilhos. R. Bras. Zootec., 26(4):787-796.

FEIJÓ, G.L.D., SILVA, J.M., THIAGO, L.R.L. et al. Efeito dos níveis de concentrado na engorda de bovinos confinados. Desempenho de novilhas nelore. In: REUNIÃO ANUAL DA SOCIEDADE BRASILEIRA DE ZOOTECNIA, 33, 1996. Fortaleza. Anais... Fortaleza: SBZ, 1996. p.70.

FOX, D.G., BLACK, J.R. 1984. A system for predicting body composition and performance of growing cattle. J. Anim. Sci., 58(3):725-739.

GOMES, B.V., QUEIROZ, A.C., FONTES, C.A.A. et al. 1994. Estudo das características físico-químicas de fenos e palha. 1. Efeitos sobre a ingestão, digestibilidade aparente e taxa de passagem da materia seca, pH e concentração de amônia ruminal. R. Soc. Bras. Zootec., 23(3):352-365.

HATENDI, P.R., LULENGA, F.M., SIBANDA, S. et al. 1996. The effect of diet and frequency of watering on the performance of growing cattle given food at maintenance. J. Anim. Sci, 63(1):33-38.

HIRONAKA, R., FREEZE, B., KOZUB, G.C. et al. 1994. Influence of barley silage:concentrate ratio on rate and efficiency of live weight gain, diet digestibility and carcass characteristics of beef steers. Can. J. Anim. Sci., 74(3):315-325.

LIMA, F.C. Digestão total e parcial da energia e proteína em taurinos, zebuínos, seus mestiços e em bubalinos. Viçosa, MG: UFV, 1986. 120p. Tese (Doutorado em Zootecnia) Universidade Federal de Viçosa, 1986.

LORENZONI, W.R, CAMPOS J., GARCIA, J.A. et al. 1986. Ganho de peso, eficiência alimentar e qualidade de carcaça de novilhos búfalos, nelores, holandeses e mestiços holandêszebu. R. Soc. Bras. Zootec., 25(6):486-497.

McDONALD, P., EDWARDS, R., GREENHALGH, J.F.D. 1993. Nutrition animal. 4.ed. Zaragoza: Acribia. 571p.

MILLER, B.G., MUNTIFERING, R.B. 1985. Production research papers. Effect of forage: concentrate on kinetics of forage fiber digestion in vivo. J. Dairy Sci., 68(1):40-44.

MONTGOMERY, M.J.. BAUMGARDT. 1965. Regulation of feed intake in ruminants, II. Rations varying in energy concentration and physical form. J. Dairy Sci., 48(12):1623-1628.

MORAN, J.B. 1985.Comparative performance of five genotypes of Indonesian large ruminants. I. Effect of dietary quality on live weight and feed utilization. Aust. J. Agric. Res., 36(5):743-752.

NATIONAL RESEARCH COUNCIL - NRC. 1984. Nutrient requirements of beef cattle. 6.rev.ed. Washington: National Academy Press. 90p.
NATIONAL RESEARCH COUNCIL - NRC. 1989. Nutrient requirements of dairy cattle. 6.rev.ed. Washington, D.C.: National Academy Press. 157p.

NATIONAL RESEARCH COUNCIL - NRC. 1996. Nutrients requirements of beef cattle. 7 th. rev. ed. Washington, D.C.: National Academy Press. 242p.

OLIVEIRA, M.A.T., FONTES, C.A.A., LANA, R.P. et al. 1994. Consumo alimentar e digestibilidade de rações com dois níveis de concentrado em bovinos de cinco grupos genéticos. R. Soc. Bras. Zootec, 23(4):667-677.

PRESTON, T.R., WILLIS, M.B. 1974. Intensive beef production. 2.ed. Oxford: Pergamon Press. 546p.

RESENDE, K.T. Digestão total e parcial da energia em bubalinos, taurinos, zebuínos e cruzamento (1/2; 3/4 e 5/8) taurinos por zebuínos. Viçosa, MG: UFV, 1984.36p. Dissertação (Mestrado em Zootecnia) - Universidade Federal de Viçosa, 1984.

RESENDE, F.D., QUEIROZ, A.C., OLIVEIRA, J.V. et al. 2001. Bovinos mestiços confinados alimentados com diferentes proporções de volumoso: concentrado. 2. Efeito sobre a ingestão dos nutrientes. Rev. bras. zootec., 30(1):264-272.

RESENDE, F.D., QUEIROZ, A.C., FONTES, C.A.A. et al. 1994. Rações com diferentes níveis de fibra em detergente neutro na alimentação de bovídeos em confinamento. R. Soc. Bras. Zootec., 23(3):366-376.

RIBEIRO,T.R. Desempenho e qualidade da carcaça de bezerros holandeses alimentados com dietas contendo diferentes níveis de concentrado. Viçosa, MG: UFV, 1997. Dissertação (Mestrado em Zootecnia) - Universidade Federal de Viçosa, 1997.

RODE, L.M., WEAKLEY, D.C., SATTER, L.D. 1985. Effect of forage amount and particle size in diets of lactating dairy cows on site of digestion and microbial syntesis. Can. J. Anim. Sci., 65(1):101-111.

RODRIGUES, L.R.R., FONTES, C.A.A., JORGE, A.M. et al. 1996. Consumo de rações contendo quatro níveis de concentrado por bovinos holandeses e nelore e por bubalinos. R. Soc. Bras. Zootec., 25(3):568-581.

ROHR, K., DAENICKE, R. 1984. Nutritional effects on the distribution of live weight as gastrointestinal tract fill and tissue components in growing cattle. J. Anim. Sci., 58(3):753-795.

STATISTICAL ANALYSIS SYSTEMS - SAS. 1992. User's Guide. Versão 6.0. Cary, NC: SAS Institute Inc. 1042p.

SILVA, D.J. 1990. Análise de alimentos (Métodos químicos e biológicos). 2.ed., Viçosa: UFV. 165p.

VALADARES FILHO, S.C., COELHO DA SILVA, J.F., LEÃO, M.I. 1987. Estudo comparativo da digestão de matéria seca e carboidratos em bovinos e bubalinos alimentados com diferentes rações. R. Soc. Bras. Zootec., 16(2):120-130.

VAN SOEST, P.J. 1994. Nutritional ecology of the ruminant. 2.ed. London: Comstock Publishing Associates. 476p.

WOODY, H.D., FOX, D.G., BLACK, J.R.1983. Effect of diet grain content on performance of growing and finishing cattle. J. Anim. Sci., 57(3):719-728.

Recebido em: 28/02/00

Aceito em: 21/09/00 